\title{
Differentially expressed TUG1 and miR-145-5p indicate different severity of chronic heart failure and predict 2-year survival prognosis
}

\author{
QINWEI ZHU, SHUANGHONG LI, KUI JI, HONGYUAN ZHOU, CHUANCHAO LUO and YANA SUI
}

Department of Emergency, Weifang Hospital of Traditional Chinese Medicine, Weifang, Shandong 261041, P.R. China

Received June 7, 2021; Accepted September 2, 2021

DOI: $10.3892 /$ etm.2021.10796

\begin{abstract}
Long non-coding RNAs (lncRNAs) and microRNAs (miRs) have critical roles in the progression of various diseases. The present study aimed to investigate the levels and clinical significance of lncRNA taurine upregulated gene 1 (TUG1) and miR-145-5p in patients with chronic heart failure (CHF) and explore their indicative value regarding disease severity. TUG1 and miR-145-5p levels were detected by reverse-transcription quantitative PCR. Correlations were examined using Pearson's correlation analysis. Receiver operating characteristic analysis was used to evaluate the diagnostic value of TUG1, miR-145-5p and brain natriuretic peptide (BNP). Survival analysis was performed by the Kaplan-Meier method. Cox regression analysis was used to evaluate the prognostic value of TUG1 and miR-145-5p. The levels of interleukin- 6 and tumor necrosis factor- $\alpha$ in serum were detected by ELISA. The results indicated that TUG1 was upregulated and miR-145-5p was downregulated in patients with CHF and they were negatively correlated. TUG1 and miR-145-5p were associated with the left ventricle ejection fraction and were able to indicate the severity of CHF. Serum TUG1 and miR-145-5p had a certain diagnostic value and the combination of BNP, TUG1 and miR-145-5p had high diagnostic accuracy. TUG1 and miR-145-5p were closely associated with overall survival and may function as independent prognostic biomarkers for patients with CHF. In addition, TUG1 and miR-145-5p levels were markedly correlated with inflammation in CHF. Upregulated TUG1 and downregulated miR-145-5p may indicate the severity of CHF, may serve as diagnostic and prognostic biomarkers and may be involved in $\mathrm{CHF}$ progression by regulating inflammatory responses.
\end{abstract}

Correspondence to: Dr Yana Sui, Department of Emergency, Weifang Hospital of Traditional Chinese Medicine, 1055 Weizhou Road, Kuiwen, Weifang, Shandong 261041, P.R. China

E-mail: suiyana_wfzy@163.com

Key words: TUG1, miR-145-5p, chronic heart failure, severity, diagnosis, prognosis, inflammation

\section{Introduction}

Heart failure (HF) is the end stage of various heart diseases (1) and is caused by myocardial injury due to incidences such as myocardial infarction, cardiomyopathy, hypertension and inflammation (2). In addition, HF is one of the leading causes of human morbidity and mortality in the world (3). Patients with HF usually have adverse symptoms such as dyspnea, peripheral edema and ascites, fatigue and nausea (2). HF is usually a chronic condition referred to as chronic HF (CHF), which may be stabilized, worsened or decompensated (4). The basic etiology of CHF is mainly the reduction and damage of cardiomyocytes. Due to the occurrence of myocardial tissue injury, the level of C-reactive protein (CRP, a systemic biomarker of inflammation) increases and the inflammatory response is also activated $(5,6)$. Brain natriuretic peptide (BNP) testing has been proposed as a diagnostic and prognostic tool for congestive HF (7). However, it may also exhibit marked changes in other diseases, such as cirrhosis or renal failure (3). The left ventricle ejection fraction (LVEF) may be used to reflect the level of cardiac function in patients with HF (8), but not all patients with HF exhibit abnormal LVEF. Thus, exploring the key molecules involved in the pathological mechanisms of CHF will hopefully provide more effective biomarkers for this disease progression.

Non-coding RNAs, including long non-coding RNAs (lncRNAs, longer than 200 nucleotides) and microRNAs (miRs, 19-25 nucleotides long), are able to regulate the initiation and progression of various diseases, including cardiovascular diseases such as $\mathrm{CHF}$ (9). The clinical value of various IncRNAs and miRs in CHF has been established. For instance, lncRNA ZNFX1 antisense RNA1 and miR-590-3p may be used as novel markers for the diagnosis and prognosis of patients with CHF (10). Aberrant lncRNA nuclear-enriched abundant transcript 1 and miR-129-5p have been indicated to serve as novel diagnostic and prognostic markers for patients with CHF (11). A previous study reported that lncRNA taurine upregulated gene 1 (TUG1) is able to regulate hypoxia-induced cardiomyocyte injury by binding to miR-145-5p (12). In addition, TUG1 may promote cardiac hypertrophy, which is one of the major risk factors for the development of CHF (13). miR-145-5p, which is directly regulated by TUG1, is similarly closely associated with cardiomyocyte injury and CHF (14). Furthermore, miR-145-5p, an important inflammation-regulating miR, is 
able to alleviate hypoxia-induced inflammatory response in cardiac cells $(15,16)$. Similarly, TUG1 is an inflammation-related non-coding RNA that is involved in the initiation and development of inflammatory responses in a variety of diseases, or regulates the process of inflammatory responses in diseases (17-19). Therefore, the above-mentioned multiple lines of evidence suggest that the TUG1/miR-145-5p signaling axis is highly likely to be involved in CHF progression. However, the clinical value and significance of the expression levels of elements of this signaling axis in patients with $\mathrm{CHF}$ have remained elusive.

The purpose of the present study was to measure the levels of TUG1 and miR-145-5p in patients with CHF, investigate the correlation between the TUG1/miR-145-5p interaction and the severity of $\mathrm{CHF}$, evaluate the diagnostic and prognostic value of the TUG1/miR-145-5p interaction and finally investigate the correlation between the TUG1/miR-145-5p interaction and the levels of inflammatory markers. The present study may provide new and effective biomarkers for indicating the severity of $\mathrm{CHF}$ and for the diagnosis and prognosis of $\mathrm{CHF}$.

\section{Materials and methods}

Study population. This study was approved by the Ethics Committee of Weifang Hospital of Traditional Chinese Medicine (Weifang, China; approval no. 0015893). A total of 98 patients with CHF were selected as the study group for the present study, who were admitted to Weifang Hospital of Traditional Chinese Medicine (Weifang, China) between March 2016 and December 2019. The diagnosis of patients with CHF was in accordance with the criteria listed in the 2013 American College of Cardiology Foundation/American Heart Association Guidelines and the 2016 European Society of Cardiology Guidelines $(20,21)$. The inclusion criteria for patients with $\mathrm{CHF}$ were as follows: a) Clinically diagnosed CHF; b) clinically stable; c) LVEF <40\%; d) New York Heart Association (NYHA) class from II to IV; and e) no history of surgery within 1 year. Participants were excluded from the present study if they had any of the following: a) Malignancy; b) infectious disease; c) history of cerebrovascular events within 6 months; d) cardiac assist device; e) history of organ transplantation; or f) liver or kidney failure. In addition, 86 participants without $\mathrm{CHF}$ and diseases listed in the exclusion criteria, who underwent physical examination at the same hospital during the same time period, were recruited to serve as a control group. Prior to enrolment in the study, every participant was informed and signed an informed consent form.

Serum sample collection. Venous blood samples from all participants were drawn into EDTA anticoagulant tubes. Subsequently, the blood samples were immediately centrifuged at $1,500 \mathrm{x} \mathrm{g}$ at $4^{\circ} \mathrm{C}$ to obtain serum, which was stored at $-80^{\circ} \mathrm{C}$ for further analysis.

Follow-up of patients for survival. All patients with $\mathrm{CHF}$ were treated with conventional medications such as diuretics, angiotensin receptor blockers and beta blockers or angiotensin-converting enzyme inhibitors. Patients with CHF were followed up monthly by telephone for 2 years and survival information was recorded and analyzed.
$R N A$ extraction and reverse transcription-quantitative PCR $(R T-q P C R)$. Total RNA was extracted from serum with TRIzol ${ }^{\circledR}$ reagent (Invitrogen; Thermo Fisher Scientific Inc.). A NanoDrop 2000 (Thermo Fisher Scientific, Inc.) was used to analyze the concentration and quality of total RNA. The cDNA was synthesized from the obtained RNA using the PrimeScript RT reagent kit (Takara Bio Inc.).

TUG1 and miR-145-5p expression levels were measured using real-time qPCR, performed with a SYBR Green I Master Mix kit (Invitrogen; Thermo Fisher Scientific Inc.) on a 7500 Real-Time PCR System (Applied Biosystems; Thermo Fisher Scientific Inc.). All procedures were performed following the manufacturer's protocol. The following thermocycling conditions were used for qPCR: $95^{\circ} \mathrm{C}$ for $10 \mathrm{~min}$, followed by $95^{\circ} \mathrm{C}$ for $20 \mathrm{sec}, 60^{\circ} \mathrm{C}$ for $15 \mathrm{sec}$ and $72^{\circ} \mathrm{C}$ for $20 \mathrm{sec}$, for a total of 40 cycles. The expression levels of TUG1 and miR-145-5p were normalized to GAPDH and U6, respectively. The primer sequences were designed by Sangon Biotech and were as follows: TUG1 forward, 5'-TAGCAGTTCCCCAATCCTTG-3' and reverse, 5'-CACAAA TTCCCATCATTCCC-3'; GAPDH forward, 5'-CAAGGTCA TCCATGACAACTTTG-3' and reverse, 5'-GTCCACCACC CTGTTGCTGTAG-3'; miR-145-5p forward, 5'-GCCGAGG TCCAGTTTTCCCA-3' and reverse, 5'-CTCAACTGGTGT CGTGGA-3'; U6 forward, 5'-CTCGCTTCGGCAGCACA-3' and reverse, 5'-AACGCTTCACGAATTTGCGT-3'. The relative levels of TUG1 and miR-145-5p were calculated using the $2^{-\Delta \Delta C \mathrm{C}}$ method (22).

Evaluation of inflammatory markers. The levels of inflammatory markers, including CRP, interleukin (IL)-6 and tumor necrosis factor (TNF)- $\alpha$, were detected in the serum of patients with $\mathrm{CHF}$ to evaluate the inflammatory response. CRP levels were measured using an access automatic analyzer (Beckman Coulter). Human IL- 6 and TNF- $\alpha$ ELISA kits (BD Biosciences) were used to measure the levels of pro-inflammatory factors IL-6 (cat. no. 555220) and TNF- $\alpha$ (cat. no. 555212). The 96-well plates coated with highly purified antibodies (capture antibodies) for IL-6 dilution or TNF- $\alpha$ dilution, were incubated overnight at $4^{\circ} \mathrm{C}$ and then filled with dilution buffer, followed by incubation for $1 \mathrm{~h}$. Subsequently, standard or experimental samples were added into the wells and incubated at room temperature for $12 \mathrm{~h}$. Corresponding biotin-conjugated antibodies (detection antibodies) for IL-6 dilution and TNF- $\alpha$ dilution, avidin horseradish peroxidase and tetramethylbenzidine solution were added. Finally, the reaction was stopped by adding $1 \mathrm{~mol} / 1 \mathrm{H}_{3} \mathrm{PO}_{4}$. The optical density (OD) value at $450 \mathrm{~nm}$ was read with a microplate reader (Bio-Rad Laboratories, Inc.) and the concentration of the cytokines was quantified using the OD value and the standard curves.

Statistical analysis. Values are presented as the mean \pm standard deviation or the median (interquartile range). The StarBase platform (http://starbase.sysu.edu.cn/) (23) was used to predict the complementary binding sites of TUG1 with miR-145-5p. For the measurement data, an unpaired Student's t-test was used to compare differences between two groups, while differences among multiple groups were compared using one-way ANOVA followed by Tukey's post-hoc test. Comparison between categorical variables was performed by the Chi-square 
Table I. Baseline characteristics of the study population.

\begin{tabular}{|c|c|c|c|c|c|}
\hline Characteristic & $\begin{array}{l}\text { Upper } \\
\text { limit }\end{array}$ & $\begin{array}{l}\text { Controls } \\
(\mathrm{n}=86)\end{array}$ & Upper limit & $\begin{array}{c}\mathrm{CHF} \\
\text { patients }(\mathrm{n}=98)\end{array}$ & P-value \\
\hline Age, years & 77 & $65.861 \pm 5.166$ & 76 & $64.867 \pm 4.800$ & 0.178 \\
\hline Male sex & - & $49(56.98)$ & - & $58(59.18)$ & 0.762 \\
\hline BMI, $\mathrm{kg} / \mathrm{m}^{2}$ & 26.83 & $25.012 \pm 0.632$ & 26.66 & $25.156 \pm 0.656$ & 0.133 \\
\hline Smoking & - & $50(58.14)$ & - & $59(60.20)$ & 0.776 \\
\hline Alcohol abuse, & - & $52(60.47)$ & - & $63(64.29)$ & 0.593 \\
\hline Hypertension & - & $52(60.47)$ & - & $66(67.35)$ & 0.332 \\
\hline Diabetes & - & $49(56.98)$ & - & $57(58.16)$ & 0.871 \\
\hline $\mathrm{TC}, \mathrm{nM}$ & 5.10 & $4.660 \pm 0.205$ & 5.08 & $4.686 \pm 0.179$ & 0.346 \\
\hline $\mathrm{TG}, \mathrm{nM}$ & 2.56 & $1.355 \pm 0.539$ & 2.61 & $1.413 \pm 0.552$ & 0.469 \\
\hline HDL-C, nM & 1.81 & $1.214 \pm 0.275$ & 1.85 & $1.167 \pm 0.185$ & 0.171 \\
\hline LDL-C, nM & 3.69 & $2.876 \pm 0.172$ & 3.45 & $2.935 \pm 0.268$ & 0.082 \\
\hline BNP ng/1 & 161.60 & $61.788 \pm 26.847$ & $3,377.06$ & $1,470.179 \pm 796.906$ & $<0.001$ \\
\hline $\mathrm{LVEF}, \%$ & 64.54 & $59.773 \pm 1.824$ & 34.41 & $22.038 \pm 6.087$ & $<0.001$ \\
\hline CRP, mg/l & 14.33 & $7.642 \pm 2.621$ & 36.56 & $21.164 \pm 7.632$ & $<0.001$ \\
\hline \multicolumn{6}{|l|}{ NYHA class } \\
\hline II & - & - & - & 48 & - \\
\hline III & - & - & - & 27 & - \\
\hline IV & - & - & - & 23 & - \\
\hline
\end{tabular}

Values are expressed as $\mathrm{n}, \mathrm{n}(\%)$ or the mean \pm standard deviation. CHF, chronic heart failure; BMI, body mass index; TC, total cholesterol; TG, triglyceride; HDL-C, high-density lipoprotein cholesterol; LDL-C, low-density lipoprotein cholesterol; BNP, brain natriuretic peptide; LVEF, left ventricle ejection fraction; CRP, C-reactive protein; NYHA, New York Heart Association.

test. Pearson correlation analysis was used to evaluate the correlation between different variables. The diagnostic value of TUG1, miR-145-5p and BNP for screening patients with CHF was assessed by receiver operating characteristic (ROC) curve analysis. Kaplan-Meier survival analysis and log-rank tests were used to identify the association of TUG1 and miR-145-5p expression with overall survival of patients with CHF. The prognostic value of TUG1 and miR-145-5p for patients with CHF was confirmed by Cox regression analysis. SPSS 22.0 (IBM Corp.) and GraphPad Prism 7.0 software (GraphPad Software, Inc.) were used to perform the statistical analysis. Each experiment was repeated at least 3 times. $\mathrm{P}<0.05$ was considered to indicate statistical significance.

\section{Results}

Baseline characteristics of the study population. A total of 98 patients with $\mathrm{CHF}$ and 86 controls were recruited for the present study. The baseline characteristics of all participants are presented in Table I. There were no significant differences in age, sex, body mass index, smoking, alcohol abuse, hypertension, diabetes, total cholesterol, triglyceride, high-density lipoprotein cholesterol or low-density lipoprotein cholesterol (all $\mathrm{P}>0.05$ ) between patients with $\mathrm{CHF}$ and controls. However, the group of patients with $\mathrm{CHF}$ was indicated to have significantly higher BNP and CRP levels and significantly lower LVEF levels than the control group (all $\mathrm{P}<0.001$ ). Furthermore, the number of patients with CHF with NYHA class II, III and IV was 48, 27 and 23, respectively.
Differential expression of TUG1 and miR-145-5p in patients with CHF. Complementary binding sites between TUG1 and miR-145-5p were predicted with the StarBase platform (Fig. 1A), which indicated a direct interaction at the nucleic acid level. Compared with the controls, relative TUG1 expression was increased $(\mathrm{P}<0.001$; Fig. $1 \mathrm{~B})$ and relative miR-145-5p expression was decreased $(\mathrm{P}<0.001$; Fig. 1C) in patients with CHF. As indicated in Fig. 1D, the relative expression of TUG1 was negatively correlated with the relative expression of miR-145-5p in CHF patients $(r=-0.809, \mathrm{P}<0.001)$.

Expression comparison of the TUG1/miR-145-5p interaction between patients with $\mathrm{CHF}$ with different severity. The results of the correlation analysis of TUG1 or miR-145-5p with LVEF demonstrated a significant negative correlation between TUG1 and LVEF ( $\mathrm{r}=-0.554, \mathrm{P}<0.001$; Fig. $2 \mathrm{~A}$ ) and a significant positive correlation between miR-145-5p and LVEF $(r=0.377$, $\mathrm{P}<0.001$; Fig. 2B). TUG1 and miR-145-5p expression levels were then analyzed in patients with different NYHA class. The expression level of TUG1 was upregulated with increasing NYHA class (all $\mathrm{P}<0.001$; Fig. 2C), while the expression level of miR-145-5p was downregulated with increasing NYHA class (all $\mathrm{P}<0.001$; Fig. 2D).

Diagnostic value of the TUG1/miR-145-5p interaction and $B N P$ in patients with $C H F$. The results of the ROC analyses are presented in Fig. 3. BNP is one of the key molecules that has been proved to be closely associated with the occurrence and development of CHF. Thus, ROC analysis was 
B

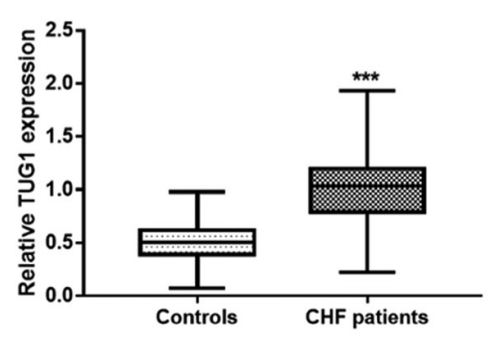

C

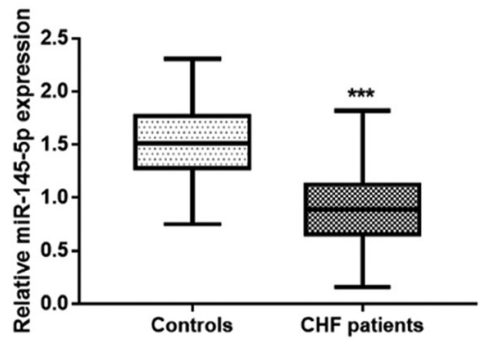

D

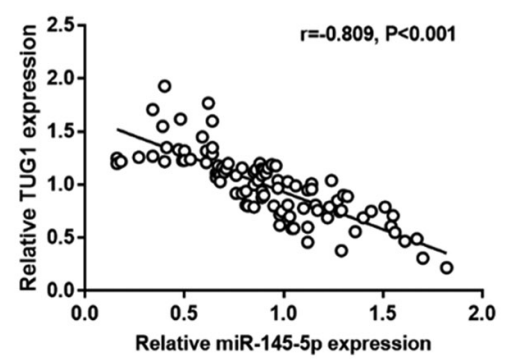

Figure 1. Aberrant expression of TUG1 and miR-145-5p in patients with CHF. (A) Binding sites of TUG1 with miR-145-5p. (B) Relative TUG1 expression in patients with CHF and controls. (C) Relative miR-145-5p expression in patients with CHF and controls. (D) Negative correlation between TUG1 and miR-145-5p expression ( $\mathrm{r}=-0.809, \mathrm{P}<0.001) .{ }^{* * *} \mathrm{P}<0.001$ vs. Controls. The values are expressed as the median (interquartile range) in the box plots. TUG1, taurine upregulated gene 1 ; miR, microRNA; $\mathrm{CHF}$, chronic heart failure.
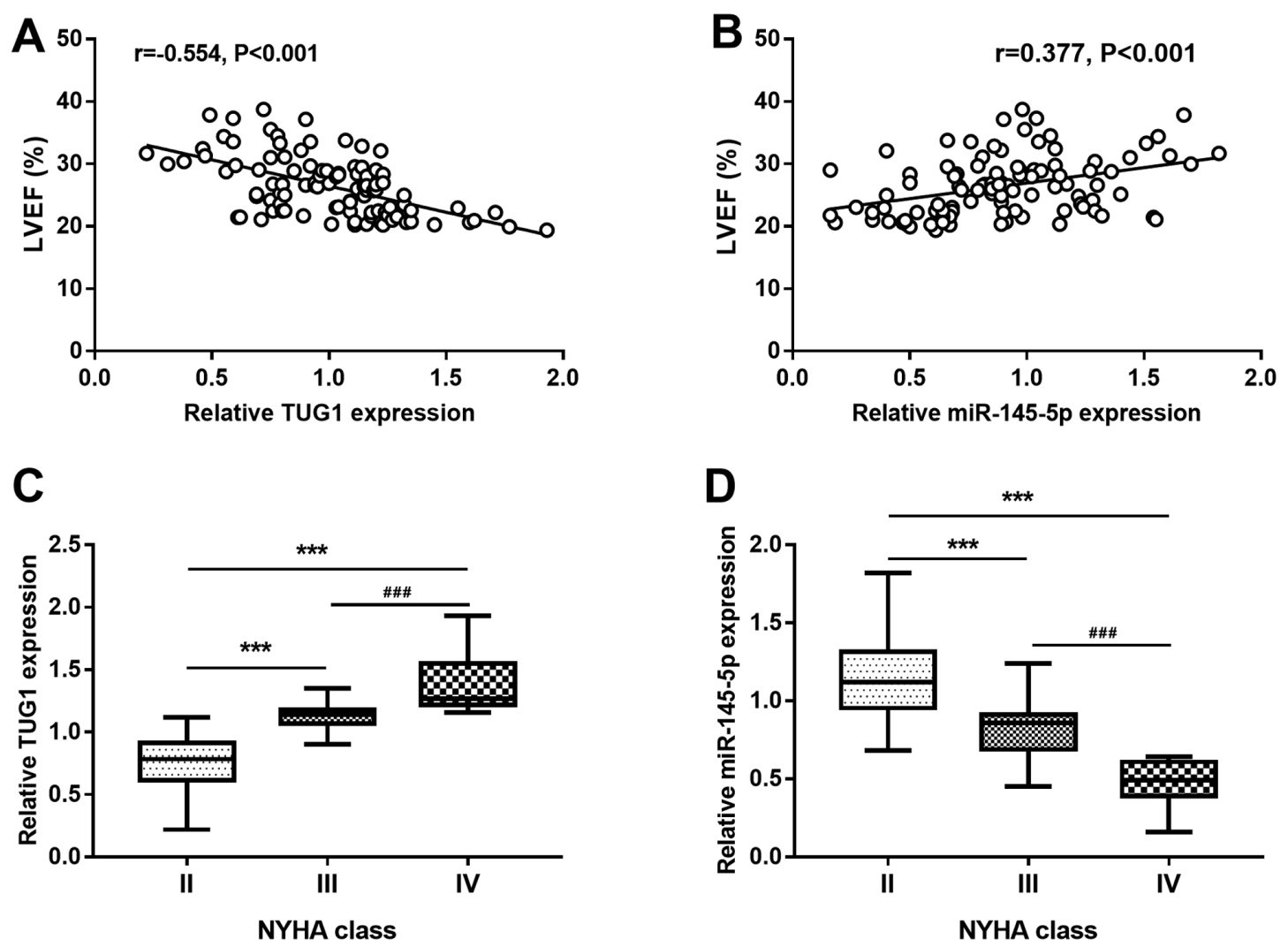

Figure 2. Correlation of the TUG1/miR-145-5p interaction with LVEF and severity of CHF in patients. (A) Negative correlation between relative TUG1 expression and LVEF ( $\mathrm{r}=-0.554, \mathrm{P}<0.001$ ). (B) Positive correlation between relative miR-145-5p expression and LVEF ( $\mathrm{r}=0.377, \mathrm{P}<0.001)$. (C) Relative TUG1 expression in patients with CHF with NYHA class II, III and IV. (D) Relative miR-145-5p expression in patients with CHF with NYHA class II, III and IV. ${ }^{* * *}$ P $<0.001$ vs. patients with NYHA class II; ${ }^{\# \#} \mathrm{P}<0.001$ vs. patients with NYHA class III. The values are expressed as the median (interquartile range) in the box plots. TUG1, taurine upregulated gene 1; miR, microRNA; LVEF, left ventricle ejection fraction; NYHA, New York Heart Association; CHF, chronic heart failure.

performed to evaluate its value in diagnosing $\mathrm{CHF}$ and the area under the curve (AUC) was 0.859 [95\% confidence interval $(\mathrm{CI})=0.797-0.922]$. In addition, ROC analysis of TUG1 and miR-145-5p was also performed. TUG1 had a high diagnostic value for differentiating CHF with an AUC of 0.924 [95\% CI=0.884-0.964]. At a cutoff value of 0.745 , the sensitivity and specificity were 81.63 and $94.19 \%$, respectively. The diagnostic accuracy of miR-145-5p was higher than that of BNP with an AUC of 0.898 (95\% CI=0.854-0.942). Furthermore, the combination of BNP, TUG1 and miR-145-5p exhibited the highest diagnostic value (AUC $=0.991$, sensitivity $=94.90 \%$ and specificity $=98.84 \%$ at a cutoff value of 0.724 ). 


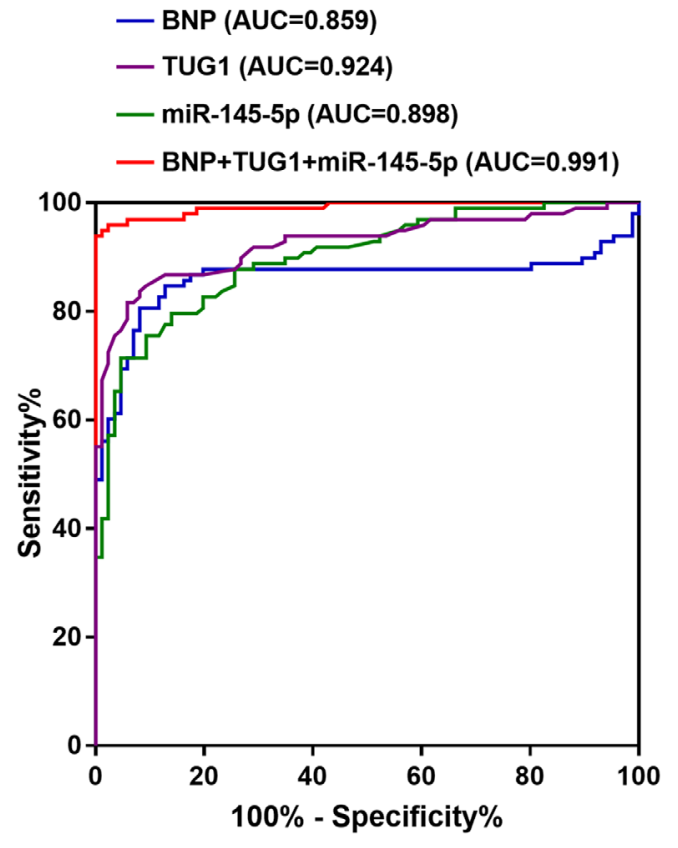

Figure 3. Diagnostic value of BNP and TUG1/miR-145-5p interaction for screening patients with CHF. BNP, brain natriuretic peptide; TUG1, taurine upregulated gene 1; miR, microRNA; AUC, area under the receiver operating characteristic curve; $\mathrm{CHF}$, chronic heart failure.

Association of the TUG1/miR-145-5p interaction with the 2-year survival of patients with CHF. The 2-year follow-up statistics indicated that $27(27.6 \%)$ patients died of cardiac disease. Of note, none of the patients were lost to follow-up during the 2-year study period. According to the median, TUG1 expression was divided into high expression (49 cases) and low expression (49 cases) and miR-145-5p was divided into high expression (49 cases) and low expression (49 cases). The results in Fig. 4A indicated that patients with low TUG1 expression had markedly higher overall survival than those with high TUG1 expression (log-rank $\mathrm{P}=0.009)$. Furthermore, the results in Fig. 4B suggested that patients with high miR-145-5p expression had significantly higher overall survival (log-rank $\mathrm{P}=0.024)$. The results of the univariate and multivariate Cox analysis are provided in Table II. The results of the univariate Cox analysis demonstrated that BNP, LVEF, NYHA class, TUG1 and miR-145-5p were significant factors associated with overall survival of patients with CHF. Significant baseline variables from the univariate analysis results were included in a subsequent multivariate analysis. BNP, LVEF and NYHA class were used to adjust for TUG1 and miR-145-5p in multivariate analysis. The multivariate analysis indicated that BNP [hazard ratio $(\mathrm{HR})=1.995,95 \% \mathrm{CI}=1.071-2.993, \mathrm{P}=0.045]$, NYHA class $(\mathrm{HR}=2.154,95 \% \mathrm{CI}=1.197-3.215, \mathrm{P}=0.023)$, TUG1 $(\mathrm{HR}=2.122$, 95\% $\mathrm{CI}=1.482-2.997, \mathrm{P}=0.003)$ and $\mathrm{miR}-145-5 \mathrm{p}(\mathrm{HR}=2.087$, $95 \% \mathrm{CI}=1.209-2.769, \mathrm{P}=0.008)$ were independently associated with overall survival of patients with CHF.

Correlation of the TUG1/miR-145-5p interaction with the levels of CRP and pro-inflammatory cytokines in CHF. The relative expression of TUG1 was positively correlated with the levels of CRP ( $r=0.557, \mathrm{P}<0.001$; Fig. 5A), IL-6 ( $\mathrm{r}=0.300, \mathrm{P}=0.003$; Fig. $5 B)$ and TNF- $\alpha(r=0.486, P<0.001$; Fig. $5 C)$. In addition, relative miR-145-5p expression was negatively correlated with
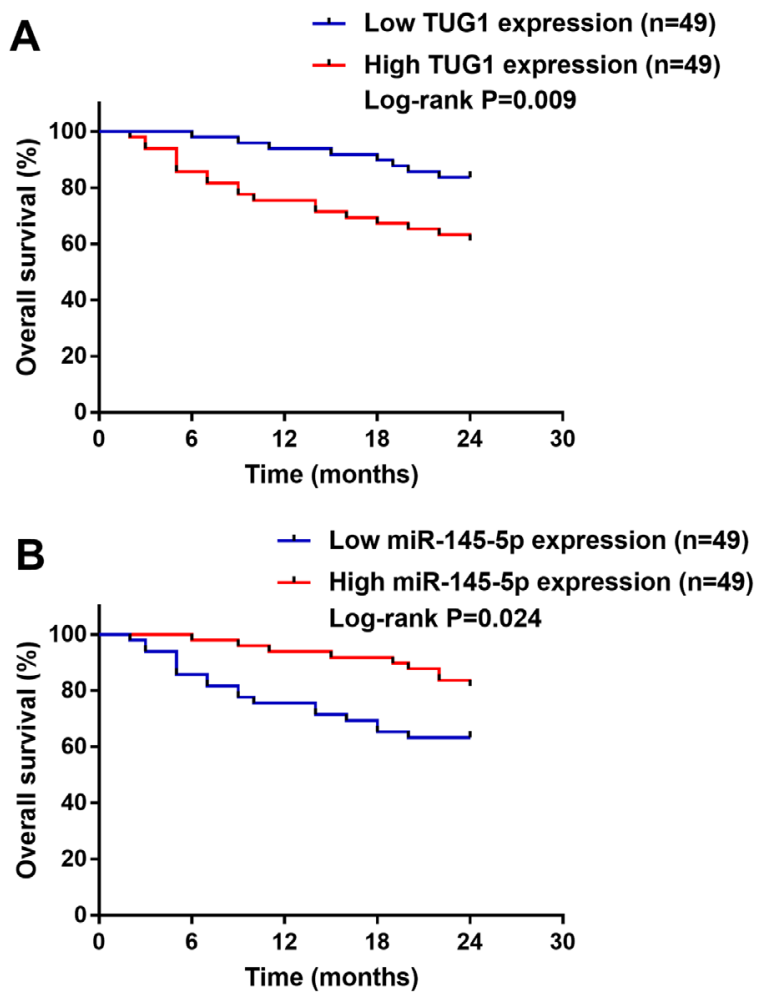

Figure 4. Relationship between TUG1/miR-145-5p interaction and survival of patients with CHF. (A) Association between TUG1 expression and overall survival of patients with CHF (log-rank P=0.009). (B) Association between miR-145-5p expression and overall survival of patients with CHF (log-rank $\mathrm{P}=0.024)$. TUG1, taurine upregulated gene 1 ; miR, microRNA; $\mathrm{CHF}$, chronic heart failure.

the levels of CRP ( $r=-0.526, P<0.001$; Fig. 5D), IL-6 ( $r=-0.267$, $\mathrm{P}=0.008$; Fig. 5E) and TNF- $\alpha(\mathrm{r}=-0.432, \mathrm{P}<0.001$; Fig. 5F).

\section{Discussion}

An increasing number of studies have indicated that lncRNAs and miRs may serve as novel targets for cardiovascular diseases, including CHF. For instance, lncRNA growth-arrest-associated (GAS) IncRNA 1 expression was reported to be decreased in patients with CHF and it may ameliorate CHF via suppressing the apoptosis of cardiomyocytes (24). The expression of miR-129-5p has been indicated to be decreased in patients with CHF and miR-129-5p may improve cardiac function in patients with CHF (25). Of note, Li et al (26) reported that GAS5 was decreased and miR-223-3p was increased in patients with CHF and reduced GAS5 had detrimental effects on CHF progression by regulating myocardial cell function via targeting miR-223-3p. The above studies demonstrated the important roles of lncRNAs and miRs in the occurrence and development of CHF. In addition, abnormal TUG1 has been reported to regulate myocardial viability and apoptosis through targeting miR-132-3p (27). TUG1 reduction improves cardiomyocyte function by regulating miR-9a-5p (28). Furthermore, numerous studies have reported the critical role of miR-145-5p in myocardial injury. For instance, Wu et al (29) suggested that miR-145-5p mimics enhanced cardiomyocyte apoptosis to regulate cardiomyocyte injury. High expression of miR-145-5p in myocardial cells promoted apoptosis of 
Table II. Cox regression analysis of factors associated with the survival of patients with chronic heart failure.

\begin{tabular}{|c|c|c|c|c|}
\hline \multirow[b]{2}{*}{ Variable } & \multicolumn{2}{|c|}{ Univariate analysis } & \multicolumn{2}{|c|}{ Multivariate analysis } \\
\hline & HR $(95 \% \mathrm{CI})$ & P-value & $\mathrm{HR}(95 \% \mathrm{CI})$ & P-value \\
\hline Age (high vs. low) & $1.121(0.712-1.526)$ & 0.552 & - & - \\
\hline Sex (male vs. female) & $1.236(0.739-1.771)$ & 0.412 & - & - \\
\hline BMI (high vs. low) & $1.328(0.854-1.855)$ & 0.185 & - & - \\
\hline Smoking (yes vs. no) & $1.227(0.829-1.607)$ & 0.224 & - & - \\
\hline Alcohol abuse (yes vs. no) & $1.410(0.821-1.978)$ & 0.371 & - & - \\
\hline Hypertension (yes vs. no) & $1.395(0.921-1.894)$ & 0.118 & - & - \\
\hline Diabetes (yes vs. no) & $1.189(0.865-1.587)$ & 0.587 & - & - \\
\hline TC (high vs. low) & $1.451(0.930-2.085)$ & 0.129 & - & - \\
\hline TG (high vs. low) & $1.519(0.942-2.161)$ & 0.098 & - & - \\
\hline HDL-C (low vs. high) & $1.392(0.926-1.973)$ & 0.110 & - & - \\
\hline LDL-C (high vs. low) & $1.618(0.984-2.335)$ & 0.074 & - & - \\
\hline BNP (high vs. low) & $2.012(1.127-2.887)$ & 0.031 & $1.995(1.071-2.993)$ & 0.045 \\
\hline LVEF (low vs. high) & $1.841(1.188-2.642)$ & 0.026 & $1.612(0.993-2.337)$ & 0.059 \\
\hline CRP (high vs. low) & $1.408(0.939-1.970)$ & 0.132 & - & - \\
\hline NYHA class (IV vs. II+III) & $2.185(1.213-3.157)$ & 0.011 & $2.154(1.197-3.215)$ & 0.023 \\
\hline TUG1 (high vs. low) & $2.305(1.508-3.207)$ & 0.002 & $2.122(1.482-2.997)$ & 0.003 \\
\hline miR-145-5p (low vs. high) & $2.229(1.373-3.083)$ & 0.005 & $2.087(1.209-2.769)$ & 0.008 \\
\hline
\end{tabular}

BMI was grouped based on the cutoff value of $24 \mathrm{~kg} / \mathrm{m}^{2}$. Age, TC, TG, HDL-C, LDL-C, BNP, LVEF, CRP, TUG1 and miR-145-5p were grouped based on their corresponding median values. Low group was defined as < cutoff value, and high group was defined as $\geq$ cutoff value. BMI, body mass index; TC, total cholesterol; TG, triglyceride; HDL-C, high-density lipoprotein cholesterol; LDL-C, low-density lipoprotein cholesterol; BNP, brain natriuretic peptide; LVEF, left ventricle ejection fraction; CRP, C-reactive protein; NYHA, New York Heart Association; TUG1, taurine upregulated gene 1; miR, microRNA; HR, hazard ratio; CI, confidence interval.

myocardial cells, which may cause myocardial injury (14). Furthermore, a study demonstrated that TUG1 upregulation promoted hypoxia-induced injury of myocardial cells via regulating miR-145-5p (12). Therefore, the TUG1/miR-145-5p interaction may be involved in myocardial cell injury and CHF progression. In the present study, upregulated TUG1 and downregulated miR-145-5p were determined in patients with CHF and a negative correlation between them was observed. In addition, TUG1 and miR-145-5p were markedly correlated with cardiac function and were able to indicate the severity of CHF in affected patients. Therefore, upregulated TUG1 and downregulated miR-145-5p are involved in the progression of $\mathrm{CHF}$ and may indicate the severity of CHF.

Advances have been made in the treatment of cardiovascular disease, but the morbidity and mortality of CHF are still increasing (30). Therefore, there is an urgent requirement for effective biomarkers for diagnosing CHF and predicting the survival prognosis of patients with $\mathrm{CHF}$, which may reduce their mortality. At present, the study of BNP in the diagnosis, treatment and prognosis of HF is attracting increasing attention from researchers (31). However, various patients with clinical symptoms of CHF have normal BNP values and certain patients have falsely elevated BNP due to heterophile antibodies interfering with immune detection, affecting the diagnostic accuracy of BNP (32). Recently, studies on the diagnostic and prognostic roles of lncRNAs and miRs for various diseases have been increasing. For instance, downregulated lncRNA-D16366 may be used as an independent indicator for the diagnosis and prognosis of hepatocellular carcinoma (33). The diagnostic and prognostic value of the lncRNA focally amplified lncRNA on chromosome 1 has been reported in cervical cancer (34). In addition, serum miR-1204 has the potential to serve as an independent diagnostic and prognostic indicator for breast cancer (35). Serum miR-629 may be a novel biomarker for the diagnosis and prognosis evaluation of pancreatic cancer (36). Therefore, lncRNAs and miRs may serve as diagnostic and prognostic biomarkers.

Numerous lncRNAs and miRs have also been reported as novel biomarkers for the diagnosis and prognosis of patients with CHF. For instance, Zhang et al (37) suggested high diagnostic and prognostic value of the lncRNA myosin heavy-chain-associated RNA transcripts for CHF. A study by Sun et al (3) revealed that 1ncRNA plasmacytoma variant translocation 1 and miR-190a-5p are two potential diagnostic biomarkers for CHF. In addition, miR-22-3p may serve as a biomarker for the prediction of adverse clinical outcomes in patients with $\mathrm{CHF}$ (38). According to the results of the present study, considering the association between TUG1/miR-145-5p dysregulation and CHF progression, TUG1/miR-145-5p may be of high clinical value. Diagnostic and prognostic value analysis of TUG1/miR-145-5p was then performed. The results of the ROC analysis indicated that the diagnostic value of TUG1 and miR-145-5p in screening patients for CHF is higher than that of BNP. In addition, the combined detection of BNP, TUG1 and miR-145-5p had the best diagnostic accuracy with an AUC of 0.991. Furthermore, the results of the Kaplan-Meier analysis 
A

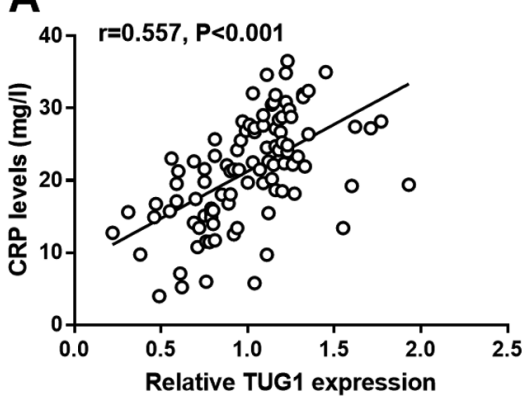

D

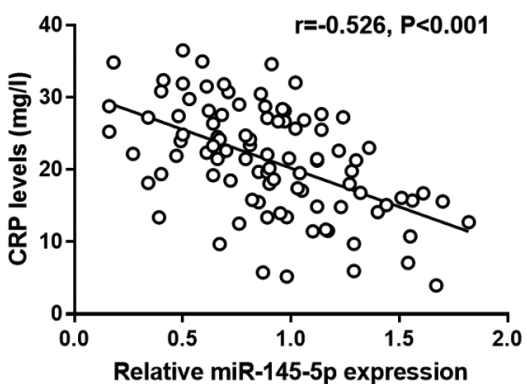

B

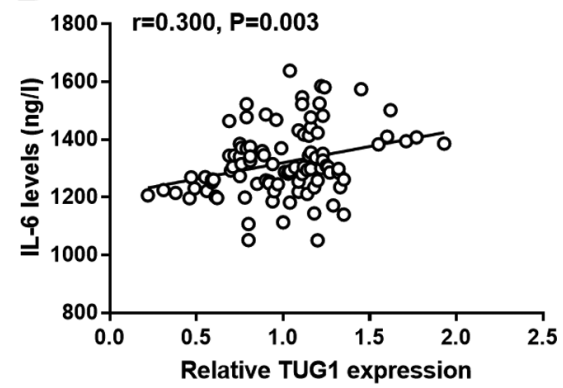

E

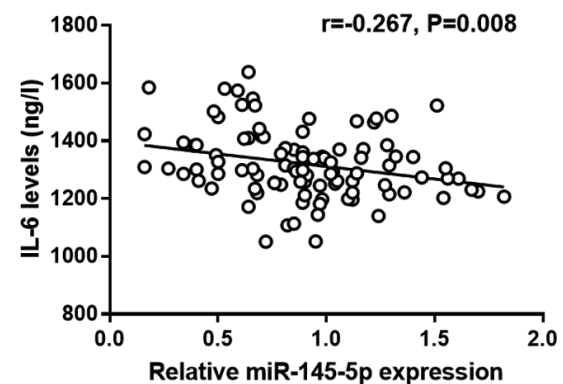

C

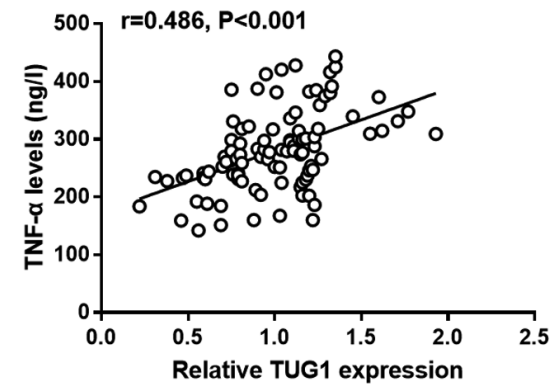

F

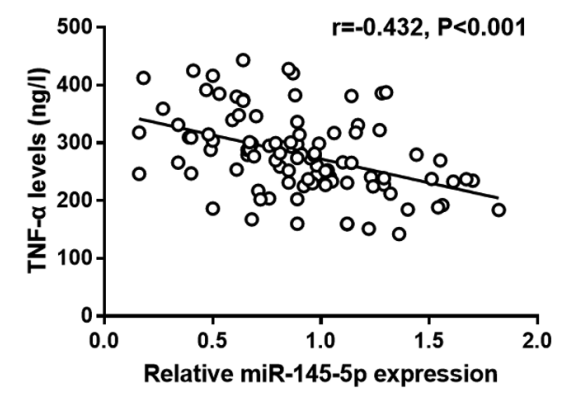

Figure 5. Correlation of TUG1/miR-145-5p interaction with the levels of CRP and pro-inflammatory cytokines in patients with CHF. (A-C) Positive correlation of TUG1 expression with (A) CRP ( $r=0.557, \mathrm{P}<0.001)$, (B) IL-6 ( $\mathrm{r}=0.300, \mathrm{P}=0.003)$ and (C) TNF- $\alpha(\mathrm{r}=0.486, \mathrm{P}<0.001)$. (D-F) Negative correlation of miR-145-5p expression with (D) CRP ( $r=-0.526, P<0.001)$, (E) IL-6 $(r=-0.267, \mathrm{P}=0.008)$ and $(\mathrm{F}) \mathrm{TNF}-\alpha(\mathrm{r}=-0.432, \mathrm{P}<0.001)$. TUG1, taurine upregulated gene 1; miR, microRNA; CHF, chronic heart failure; CRP, C-reactive protein; IL-6, interleukin-6; TNF- $\alpha$, tumor necrosis factor- $\alpha$.

suggested that high TUG1 expression and low miR-145-5p expression were significantly related to poor overall survival in patients with $\mathrm{CHF}$. The results of the Cox regression analysis indicated that the expression levels of TUG1 and miR-145-5p may be used as two independent prognostic biomarkers. Therefore, TUG1 and miR-145-5p may serve as two biomarkers for the diagnosis and prognosis of CHF.

The occurrence of myocardial inflammation in $\mathrm{CHF}$ is known to be closely associated with the progression of this disease. TUG1 and miR-145-5p, as inflammation-related non-coding RNAs, are involved in various mechanisms of disease progression by regulating inflammatory responses in numerous published studies. For instance, in a model of sepsis, TUG1 had an inhibitory effect on lipopolysaccharide (LPS)-induced inflammation of vascular endothelial cells (17). A study by Yan et al (39) revealed that miR-145-5p inhibits skin inflammatory responses in psoriasis. Of note, upregulation of TUG1 mitigated the levels of inflammatory cytokines in LPS-induced H9c2 cardiomyocytes (40). An increase in miR-145-5p has been determined to ameliorate hypoxia-induced inflammation in cardiomyocytes (15). The present study revealed the significant correlation of TUG1 and miR-145-5p with inflammatory markers (including CRP, IL- 6 and TNF- $\alpha$ ), indicating the close correlation of TUG1/miR-145-5p with inflammation in patients with CHF. Therefore, it may be concluded that the TUG1/miR-145-5p interaction may be involved in the development and progression of $\mathrm{CHF}$ through a mechanism involving the regulation of inflammatory responses, as well as the regulation of cardiomyocyte viability.

However, the present study has certain limitations. First, the sample size was small, and thus, a larger cohort is required to further validate the conclusions of the present study. In addition, the present study preliminarily explored the underlying mechanisms of the TUG1/miR-145-5p interaction involved in the progression of $\mathrm{CHF}$ by regulating myocardial inflammation, which remains to be fully explored. Thus, this mechanism should be more deeply investigated in future studies.

In conclusion, the present study demonstrated that TUG1 is upregulated and miR-145-5p is downregulated in patients with CHF and the TUG1/miR-145-5p interaction may indicate the severity of patients with CHF. In addition, dysregulated levels of TUG1 and miR-145-5p in CHF may be two biomarkers for the diagnosis and survival prognosis of $\mathrm{CHF}$, and combined detection of BNP, TUG1 and miR-145-5p may be a novel method for the diagnosis of CHF. Furthermore, the TUG1/miR-145-5p signaling interaction may be involved in the development and progression of $\mathrm{CHF}$ by regulating inflammatory responses. The TUG1/miR-145-5p interaction may also provide novel targets for the treatment of $\mathrm{CHF}$.

\section{Acknowledgements}

Not applicable.

\section{Funding}

No funding was received.

\section{Availability of data and materials}

The datasets used and/or analyzed during the current study are available from the corresponding author upon reasonable request. 


\section{Authors' contributions}

QZ, SL and YS designed the study and analyzed the clinical samples. KJ, HZ and CL collected clinical samples and follow-up information. QZ and YS analyzed the experimental data, wrote and revised the manuscript, and confirmed the authenticity of the raw data. All authors read and approved the final manuscript.

\section{Ethics approval and consent to participate}

The experimental procedures were all in accordance with the guidelines of the Ethics Committee of Weifang Hospital of Traditional Chinese Medicine and the principles of the Declaration of Helsinki, and were approved by the Ethics Committee of Weifang Hospital of Traditional Chinese Medicine (Weifang, China). Written informed consent was obtained from each patient.

\section{Patient consent for publication}

Not applicable.

\section{Competing interests}

The authors declare that they have no competing interests.

\section{References}

1. Su Q, Zhang P, Yu D, Wu Z, Li D, Shen F, Liao P and Yin G: Upregulation of miR-93 and inhibition of LIMK1 improve ventricular remodeling and alleviate cardiac dysfunction in rats with chronic heart failure by inhibiting RhoA/ROCK signaling pathway activation. Aging (Albany NY) 11: 7570-7586, 2019.

2. Kemp CD and Conte JV: The pathophysiology of heart failure. Cardiovasc Pathol 21: 365-371, 2012.

3. Sun B, Meng M, Wei J and Wang S: Long noncoding RNA PVT1 contributes to vascular endothelial cell proliferation via inhibition of miR-190a-5p in diagnostic biomarker evaluation of chronic heart failure. Exp Ther Med 19: 3348-3354, 2020.

4. Mosterd A and Hoes AW: Clinical epidemiology of heart failure. Heart 93: 1137-1146, 2007.

5. Pepys MB: The Pentraxins 1975-2018: Serendipity, diagnostics and drugs. Front Immunol 9: 2382, 2018.

6. Sun S, Ou Y, Shi H, Luo J, Luo X, Shen Y, Chen Y, Liu X, Zhu Z and Shen W: Myocardial damage associated with elective percutaneous coronary intervention in Chinese patients: A retrospective study. J Int Med Res 48: 300060520907783, 2020.

7. Carella DM: Brain Natriuretic Peptide: It's not about the brain or just another smart polypeptide - It's about the heart. Neonatal Netw 34: 355-359, 2015

8. Hiebert JB, Vacek J, Shah Z, Rahman F and Pierce JD: Use of speckle tracking to assess heart failure with preserved ejection fraction. J Cardiol 74: 397-402, 2019.

9. Wei JW, Huang K, Yang C and Kang CS: Non-coding RNAs as regulators in epigenetics (Review). Oncol Rep 37: 3-9, 2017.

10. Chang G, Zhang W, Zhang M and Ding G: Clinical value of circulating ZFAS1 and miR-590-3p in the diagnosis and prognosis of chronic heart failure. Cardiovasc Toxicol: Jul 28, 2021 (Epub ahead of print). doi: 10.1007/s12012-021-09678-7.

11. Zhang $\mathrm{H}$, Zhang $\mathrm{N}$, Jiang $\mathrm{W}$ and Lun $\mathrm{X}$ : Clinical significance of the long non-coding RNA NEAT1/miR-129-5p axis in the diagnosis and prognosis for patients with chronic heart failure. Exp Ther Med 21: 512, 2021

12. Wu Z, Zhao S, LiC and Liu C: LncRNA TUG1 serves an important role in hypoxia-induced myocardial cell injury by regulating the miR 145 5p Binp3 axis. Mol Med Rep 17: 2422-2430, 2018.

13. Zou X, Wang J, Tang L and Wen Q: LncRNA TUG1 contributes to cardiac hypertrophy via regulating miR-29b-3p. In Vitro Cell Dev Biol Anim 55: 482-490, 2019.
14. Huangfu FT, Tang LQ, Wang HQ, Zhao X and Yang M: MiR-145-5p promotes myocardial cell apoptosis in rats with myocardial infarction through PI3K/Akt signaling pathway. Eur Rev Med Pharmacol Sci 24: 12904-12911, 2020.

15. Yuan M, Zhang L, You F, Zhou J, Ma Y, Yang F and Tao L: MiR-145-5p regulates hypoxia-induced inflammatory response and apoptosis in cardiomyocytes by targeting CD40. Mol Cell Biochem 431: 123-131, 2017.

16. Li LL, Mao CD, Wang GP, Wang N and Xue AG: MiR-145-5p alleviates hypoxia/reoxygenation-induced cardiac microvascular endothelial cell injury in coronary heart disease by inhibiting Smad4 expression. Eur Rev Med Pharmacol Sci 24: 5008-5017, 2020.

17. Dong Y, Fan G, Li Y and Zhou Q: TUG1 represses apoptosis, autophagy, and inflammatory response by regulating miR-27a-3p/ SLIT2 in lipopolysaccharide-treated vascular endothelial cells. J Surg Res 256: 345-354, 2020.

18. Song B, Wu S, Ye L, Jing Z and Cao J: LncRNA TUG1 knockdown mitigates inflammatory injury induced by cigarette smoke extract in chronic obstructive pulmonary disease via miR-34c/BRD4 axis. Biosci Rep: Aug 19, 2020 (Epub ahead of print). doi: 10.1042/BSR20193896.

19. Zhao K, Tan JY, Mao QD, Ren KY, He BG, Zhang CP and Wei LZ: Overexpression of long non-coding RNA TUG1 alleviates TNF- $\alpha$-induced inflammatory injury in interstitial cells of Cajal. Eur Rev Med Pharmacol Sci 23: 312-320, 2019.

20. Yancy CW, Jessup M, Bozkurt B, Butler J, Casey DE Jr, Drazner $\mathrm{MH}$, Fonarow GC, Geraci SA, Horwich T, Januzzi JL, et al; American College of Cardiology Foundation; American Heart Association Task Force on Practice Guidelines: 2013 ACCF/AHA guideline for the management of heart failure: A report of the American College of Cardiology Foundation/ American Heart Association Task Force on Practice Guidelines. J Am Coll Cardiol 62: e147-e239, 2013.

21. Ponikowski P, Voors AA, Anker SD, Bueno H, Cleland JG, Coats AJ, Falk V, González-Juanatey JR, Harjola VP, Jankowska EA, et al; Authors/Task Force Members; Document Reviewers: 2016 ESC Guidelines for the diagnosis and treatment of acute and chronic heart failure: The Task Force for the diagnosis and treatment of acute and chronic heart failure of the European Society of Cardiology (ESC). Developed with the special contribution of the Heart Failure Association (HFA) of the ESC. Eur J Heart Fail 18: 891-975, 2016.

22. Livak KJ and Schmittgen TD: Analysis of relative gene expression data using real-time quantitative PCR and the 2(-Delta Delta C(T)) method. Methods 25: 402-408, 2001.

23. Li JH, Liu S, Zhou H, Qu LH and Yang JH: starBase v2.0: Decoding miRNA-ceRNA, miRNA-ncRNA and protein-RNA interaction networks from large-scale CLIP-Seq data. Nucleic Acids Res 42: D92-D97, 2014.

24. Deng H, Ouyang W, Zhang L, Xiao X, Huang Z and Zhu W: LncRNA GASL1 is downregulated in chronic heart failure and regulates cardiomyocyte apoptosis. Cell Mol Biol Lett 24: 41, 2019.

25. Xiao N, Zhang J, Chen C, Wan Y, Wang N and Yang J: miR-129-5p improves cardiac function in rats with chronic heart failure through targeting HMGB1. Mamm Genome 30: 276-288, 2019.

26. Li G, Du P, Qiang X, Jin D, Liu H, Li B and Guo J: Low-expressed GAS5 injure myocardial cells and progression of chronic heart failure via regulation of miR-223-3P. Exp Mol Pathol 117: 104529, 2020

27. Su Q, Liu Y, Lv XW, Dai RX, Yang XH and Kong BH: LncRNA TUG1 mediates ischemic myocardial injury by targeting miR-132-3p/HDAC3 axis. Am J Physiol Heart Circ Physiol 318: $\mathrm{H} 332-\mathrm{H} 344,2020$.

28. Yang D, Yu J, Liu HB, Yan XQ, Hu J, Yu Y, Guo J, Yuan Y and Du ZM: The long non-coding RNA TUG1-miR-9a-5p axis contributes to ischemic injuries by promoting cardiomyocyte apoptosis via targeting KLF5. Cell Death Dis 10: 908, 2019.

29. Wu G, Tan J, Li J, Sun X, Du L and Tao S: miRNA-145-5p induces apoptosis after ischemia-reperfusion by targeting dual specificity phosphatase 6. J Cell Physiol 234: 16281-16289, 2019.

30. Maggioni AP: Epidemiology of heart failure in Europe. Heart Fail Clin 11: 625-635, 2015.

31. Farnsworth CW, Bailey AL, Jaffe AS and Scott MG: Diagnostic concordance between NT-proBNP and BNP for suspected heart failure. Clin Biochem 59: 50-55, 2018.

32. Collin-Chavagnac D, Manchon M, Traulle $\mathrm{C}$ and Bernon $\mathrm{H}$ : False-positive BNP results in a 78-year-old man caused by monoclonal IgM-kappa: A case report. Clin Chim Acta 384: 179, 2007. 
33. Chao Y and Zhou D: lncRNA-D16366 is a potential biomarker for diagnosis and prognosis of hepatocellular carcinoma. Med Sci Monit 25: 6581-6586, 2019.

34. Naizhaer G, Kuerban A, Meilipa, Kuerban R and Zhou P: Up-regulation of IncRNA FALEC indicates prognosis and diagnosis values in cervical cancer. Pathol Res Pract 215: 152495, 2019.

35. Han S, Li P, Wang D and Yan H: Dysregulation of serum miR-1204 and its potential as a biomarker for the diagnosis and prognosis of breast cancer. Rev Assoc Med Bras 66: 732-736, 2020.

36. Shi W, Lu Y, Gong R, Sun JJ and Liu G: Serum miR-629 is a novel molecular marker for diagnosis and the prognosis of pancreatic cancer. Eur Rev Med Pharmacol Sci 22: 5187-5193, 2018.

37. Zhang L, Wu YJ and Zhang SL: Circulating lncRNA MHRT predicts survival of patients with chronic heart failure. J Geriatr Cardiol 16: 818-821, 2019.

38. van Boven N, Akkerhuis KM, Anroedh SS, Rizopoulos D, Pinto Y, Battes LC, Hillege HL, Caliskan KC, Germans T, Manintveld OC, et al: Serially measured circulating miR-22-3p is a biomarker for adverse clinical outcome in patients with chronic heart failure: The Bio-SHiFT study. Int J Cardiol 235: 124-132, 2017.
39. Yan JJ, Qiao M, Li RH, Zhao XT, Wang XY and Sun Q: Downregulation of miR-145-5p contributes to hyperproliferation of keratinocytes and skin inflammation in psoriasis. Br J Dermatol 180: 365-372, 2019.

40. Zhang H, Li H, Ge A, Guo E, Liu S and Zhang L: Long non-coding RNA TUG1 inhibits apoptosis and inflammatory response in LPS-treated H9c2 cells by down-regulation of miR-29b. Biomed Pharmacother 101: 663-669, 2018.

(i) $9(1)$

This work is licensed under a Creative Commons Attribution-NonCommercial-NoDerivatives 4.0 International (CC BY-NC-ND 4.0) License. 